\title{
Sustentabilidad social de agroecosistemas bovinos de doble propósito en México
}

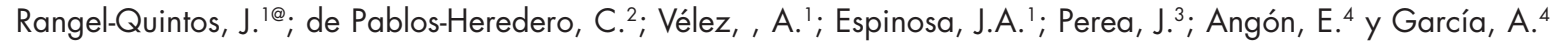

'Instituto Nacional de Investigaciones Forestales, Agrícolas y Pecuarias. INIFAP. México.

2Departamento de Economía de la Empresa, Economía Aplicada II y Fundamentos de Análisis Económico. Universidad Rey Juan Carlos.

Madrid. España.

${ }^{3}$ Universidad Técnica Estatal de Quevedo. Quevedo. Ecuador.

${ }^{4}$ Departamento de Producción Animal. Universidad de Córdoba. España.

Palabras ClaVe ADICIONALES

Sustentabilidad social.

Desarrollo humano.

Trópico húmedo.

Trópico seco.

\section{RESUMEN}

El objetivo de este estudio fue identificar las diferencias existentes en la sustentabilidad social, de los pequeños productores de ganado de doble propósito en el trópico mexicano. En dos zonas ecológicas definidas: Trópico Seco (TS) y Húmedo (TH). Para ello se analizaron las variables socioeconómicas y los indicadores de desarrollo humano establecidos por el Programa de las Naciones Unidas para el Desarrollo (PNUD). Se encontraron diferencias significativas; tanto en los objetivos de la producción (carne y leche) y en los indicadores de desarrollo humano y marginación $(p<0,05)$. Las diferencias encontradas han de ser consideradas para establecer distintas estrategias y políticas públicas para cada una de las zonas ecológicas del trópico mexicano.

\section{Agroecosystem social sustainability of dual-purpose cattle in México}

\section{SUMMARY}

The aim of this study was to identify the differences in social sustainability of smallholders of dual-purpose cattle in the Mexican tropic in two defined ecological zones: Dry tropic (TS) and humid (TH). For this, socioeconomic variables and human developing indicators (IDH) from the United Nations Development Programme (UNDP) were analyzed. Significant differences were found in both production objectives (milk and meat) and in the IDH and marginalization $(p<0.05)$. The found differences should be considered to stabilize policies and strategies publics for each ecological zones of Mexican tropic.

\section{INFORMACIÓN}

Cronología del artículo.

Recibido/received: 28.04 .15

Aceptado/Accept: 19.03.16

Online: 15.09 .16

Correspondencia a los autores/Contact e-mail:

iquintos@yahoo.com

\section{INTRODUCCIÓN}

El conocimiento de las interrelaciones entre las distintas dimensiones de la sustentabilidad técnica, económica, social y ambiental debe considerar el análisis global y el establecimiento de políticas sectoriales (Rangel-Quintos et al., 2014), debido fundamentalmente a la dualidad de objetivos y a las diferencias existentes entre el stock de recursos y sus intercambios con otros sistemas (Gallegos, 2009).

En los países en desarrollo, la sustentabilidad social (SS) apunta fundamentalmente a la satisfacción de las necesidades humanas básicas (Barbier, 1987) frente a los países desarrollados que la relacionan con conceptos de responsabilidad social (García et al., 2015). Hasta la década de los 90, la SS se percibía como un medio u obstáculo para alcanzar la sustentabilidad ecológica, asociando la pobreza y el incremento poblacional como agentes de la degradación ambiental (Foladori y Tomassino, 2000). A partir de la década del 2000, se empezó a considerar que la SS parte de la relación del ser humano con la naturaleza y, tanto la pobreza como la degradación, pueden tener una misma causa: falta de recursos o escasos derechos de propiedad. De esta forma la SS se centró en el 
incremento de las capacidades humanas. La calidad de vida pasa por tanto a ser un objetivo y no un medio para la sustentabilidad (Foladori, 2002).

El Programa de las Naciones Unidas para el Desarrollo (PNUD) plantea un enfoque sistémico donde existe una adecuada armonía de la política medioambiental con la satisfacción de las necesidades de todos, la democracia y equidad social (PNUD, 2014). Asimismo el capital humano $(\mathrm{CH})$ es el conjunto de población disponible en un territorio para el desarrollo de una actividad. Es un medio para aumentar la producción, donde la ausencia de $\mathrm{CH}$ genera un estado de vulnerabilidad creando dependencia externa y tecnológica. Por otra parte, el exceso de $\mathrm{CH}$ requerirá de un mayor potencial organizativo y de procesos de formación (García et al., 2015). Para poder evaluar la sustentabilidad de una actividad productiva en un territorio es necesario conocer cómo se desarrollan los sistemas de producción y sus efectos sobre el medio ambiente (Ramos y Garrido, 2011).

En las regiones tropicales, las unidades productivas son heterogéneas (Torres et al., 2014), pudiendo identificar agroecológicamente dos zonas claramente diferenciadas en el territorio: trópico húmedo $(\mathrm{TH})$ y trópico seco (TS), que coexisten con agricultura de temporada y con ecosistemas forestales, lo que les permite disponer de una mayor diversificación productiva (Del Castillo, 2011).

En este trabajo se profundiza en el conocimiento de la sustentabilidad social de los sistemas de doble propósito de la zona tropical de México, estableciendo las diferencias entre el trópico seco (TS) y el trópico húmedo (TH).

\section{METODOLOGÍA}

El análisis de sustentabilidad se basó en las variables socioeconómicas e indicadores de desarrollo humano propuestos por el PNUD (2014). Para ello, se utilizaron variables socioeconómicas derivadas de la información de un cuestionario aplicado a 3603 productores de ganado de doble propósito beneficiarios del programa Asistencia Técnica Pecuaria (SAGARPA-México) del ciclo 2010-2011, distribuidos en 129 municipios de 11 Estados tropicales de México (Sinaloa, Colima, Chiapas, Campeche, Michoacán, Nayarit, Tabasco, Oaxaca, Nayarit, Morelos y Veracruz). La selección se realizó por medio de muestreo no probabilístico. Para las variables de desarrollo humano se obtuvieron ocho indicadores elaborados a partir de la metodología propuesta por PNUD (2013).

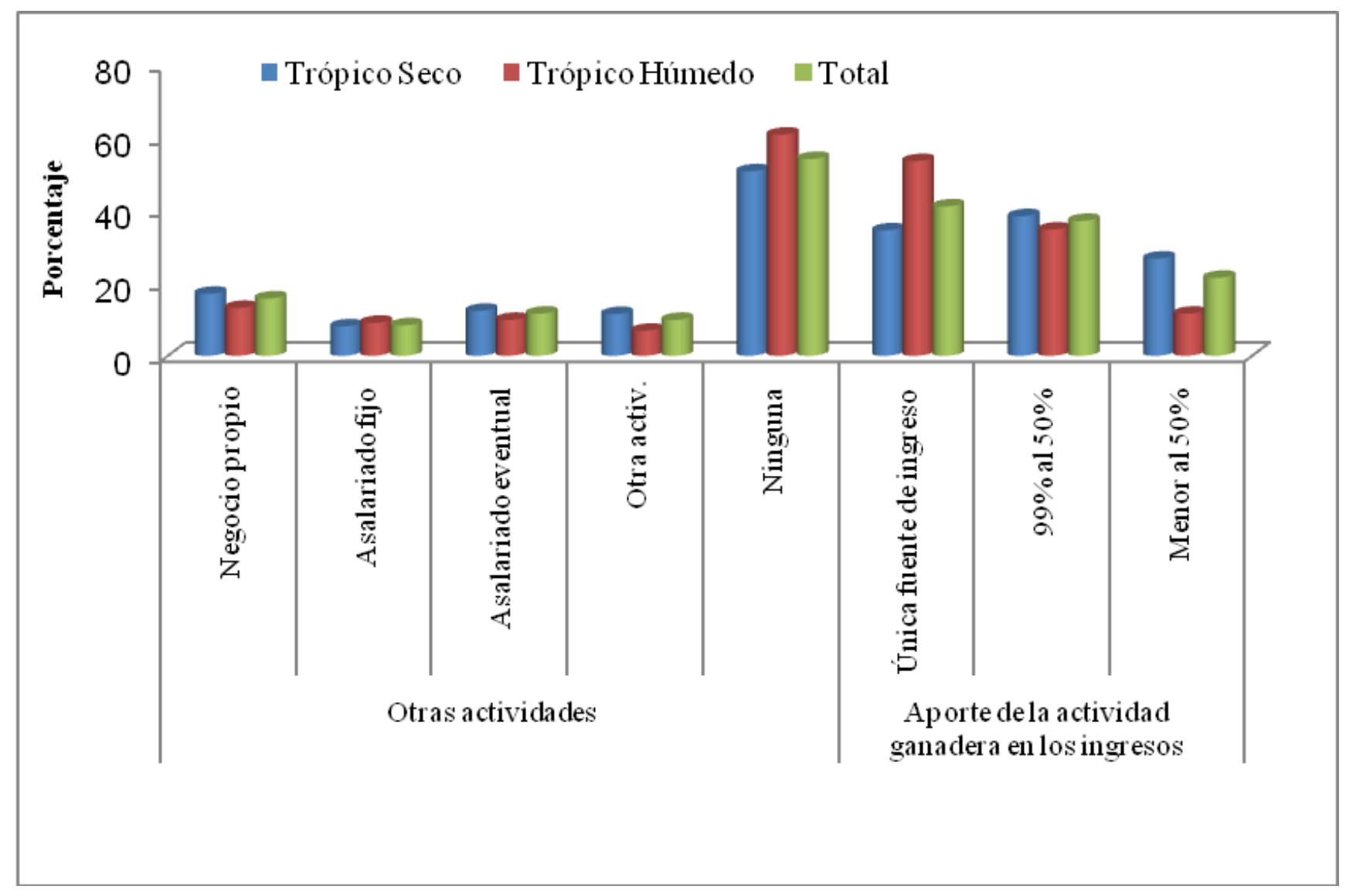

Figura 1. Importancia de la actividad ganadera en la familia, $N=3603$ (Importance of farming for the family, $N=3603$ ). 


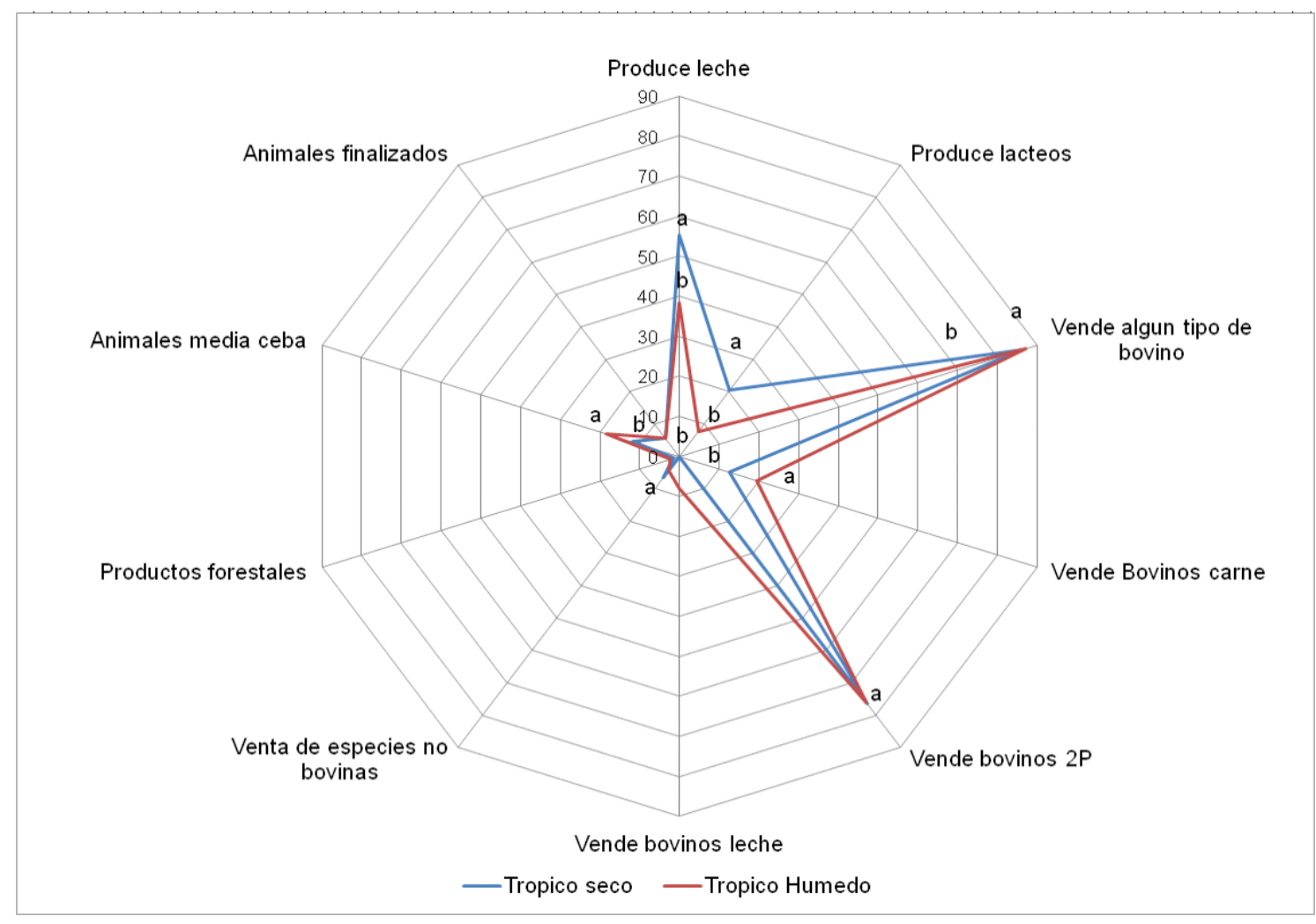

Figura 2. Porcentaje de productos comercializados en la unidad de producción ganadera; $N=3,603 ; p<0,05$ (Percentage of commercialized farm products; $\mathrm{N}=3,603 ; \mathrm{p}<0.05)$.

La muestra se segmentó a partir de la ubicación agroecológica de las explotaciones, ya sea trópico húmedo (TH) y trópico seco (TS), bajo los criterios de Torres et al. (2014) y utilizando mapas del portal web Geoinformación de la Comisión Nacional para el Conocimiento y Uso de la Biodiversidad CONABIO (2012) mediante el software ARCGIS, 9.1.

Las diferencias entre el TH y TS se analizaron con la prueba t de Student para variables cualitativas y ANOVA para cuantitativas. La información se ha tratado con el software SPSS-21.

\section{RESULTADOS}

El $65 \%$ de la muestra se localiza en el TS y el $45 \%$ en el TH. No se aprecian diferencias significativas entre las edades promedio de los productores (51 años), ni en el tiempo de duración en la asistencia técnica recibida y en dependientes familiares. Las explotaciones ubicadas en el TS generan la mitad de empleo que las del TH $(0,46$ UTA vs 0,78 UTA; $p<0,01$ ). Asimismo, la estructura es diferente. En el TS es de carácter familiar frente al TH que es mano de obra asalariada $(p<0,05)$. Asimismo en relación al género, hay una mayor participación de las mujeres en el TH (14,3\%) contra 9,9\% de TS.

En la contribución de la ganadería a los ingresos, la mayoría de las respuestas apuntan a que en el TH solo se dedican a la ganadería. Los productores de TS indican que, aparte de la explotación bovina, un 17 \% cuentan con negocio propio, y con asalariados eventuales en un $12 \%$ de lo casos. En el TH hay mayor tendencia al asalariado fijo $9 \%$. Estos resultados son coherentes con el aporte de la actividad ganadera en los ingresos ya que principalmente, los productores indican que no tienen otra actividad económica adicional y dependen mayoritariamente de la ganadería $(\mathrm{p}<0,05)$ (figura 1).

Se encontraron diferencias en el sistema de producción prevaleciente, dado que el TH es principalmente extensivo $79 \%$, mientras que el TS se ubica entre la modalidad semi extensiva $(52,5 \%)$ y la extensiva $(45,7)$. Estos resultados se relacionan directamente con la disposición de recursos forrajeros y superficie disponible.

Existen diferencias significativas en la proporción de productos generados en la explotación (figura 2). El TS es un sistema leche-carne, donde hay un mayor número de productores que generan leche y derivados, mientras que esa tendencia se modifica en el TH al tratarse de un sistema de orientación cárnica que se complementa con la producción de leche. Hay muchos factores que determinan la orientación del sistema: nivel de propagación de enfermedades en el TS o el nivel tecnológico o bien las característica del mercado de consumo.

De acuerdo a la clasificación de (PNUD, 2013) y (CONAPO, 2010), la mayoría de los territorios se clasifi- 


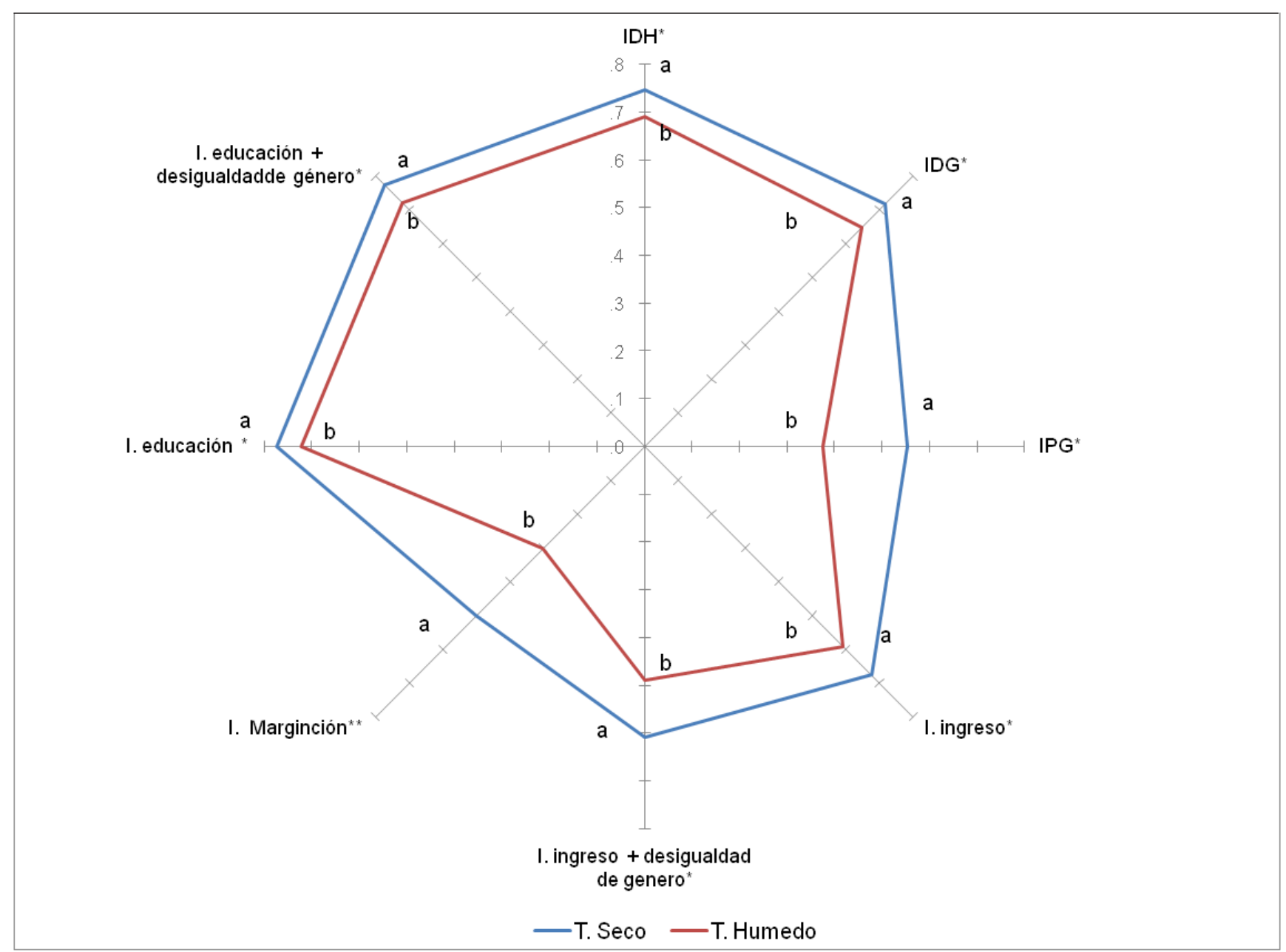

Figura 3. Indicadores de desarrollo humano y marginación (Human development and marginalization indexes). *PNUD (2013); **CONAPO (2010); $p<0,05$.

can en niveles de desarrollo medio, no obstante, se han encontrado diferencias significativas en todos los indicadores de desarrollo humano y de marginación entre los territorios (figura 3 ). Los tres indicadores con mayores diferencias entre sistemas $(\mathrm{p}<0,01)$, con valores inferiores en las explotaciones del TS son el índice de marginación, el índice de potenciación de género y el índice de ingreso más desigualdad de género. Asimismo las explotaciones ubicadas en el TH muestran los distintos indicadores de desarrollo humano por debajo del sistema del TS. Hay diferencias significativas en el ingreso per cápita por regiones, dado que en TS es de 6302 dls/año/per cápita, mientras que en TS es de 3660. La desigualdad y los desequilibrios de género implican una menor participación económica y poder para adoptar decisiones en las mujeres, especialmente en áreas económicas y políticas.

\section{CONCLUSIONES}

A pesar de que existen algunas coincidencias en cuanto al trópico húmedo y seco, se han encontrado diferencias en aspectos clave en cuanto a importancia de la actividad para los productores, venta de productos de la explotación e indicadores de desarrollo y marginación. Estas circunstancias indican potenciación y vocaciones productivas con tendencias distintas entre el Trópico Seco y Húmedo, por cuanto dichas diferencias deberían ser previstas en proyectos y programas de desarrollo donde históricamente se ha considerado a la ganadería de doble propósito con uno grupo homogénea en todo el territorio del trópico.

\section{AGRADECIMIENTO}

El estudio se ha realizado dentro del Proyecto 1-1.121541832011-P-P.1-1. La adopción y evaluación del impacto de la tecnología implementada en sistemas bovinos de doble propósito en México del Instituto Nacional de Investigaciones Forestales, Agrícolas y Pecuarias (INIFAP), México.

\section{BIBLIOGRAFÍA}

Barbier, E. 1987. The concept of sustainable economic conservation. Environ Conserv, 14: 101-110. 
CONABIO. 2012. Portal de Geoinformación, Sistema Nacional de Información sobre Biodiversidad. http://www.conabio.gob.mx/ informacion/gis/ (01/08/2014).

CONAPO. 2010. Consejo Nacional de Población. Secretaria de Gobernación. (México, Ed.). http://www.gob.mx/conapo/articulos/ 3-datos-relevantes-sobre-los-resultados-de-los-niveles-demarginacion-por-entidad-federativa-y-municipio-2015 (08/03/ 2016).

Del Castillo, P.E. 2011. El trópico mexicano: Una potencia productiva. AAPAUNAM Academia, Ciencia y Cultura, 3: 117-118.

Foladori G. y Tomassino, H. 2000. El enfoque técnico y el enfoque social de la sustentabiidad. Rev Paranaense Desenv Econ Social, 98: 67-75.

Foladori, G. 2002. Avances y límites de la sustentabilidad social. Econ Soc Territorio, 3: 621-637.

Gallegos, M. 2009. El desarrollo humano sustentable no es posible en el capitalismo. La construcción de (algunas) alternativas desde abajo. Revista Herramientas-web, debate y crítica Marxista 3. http:// www.herramienta.com.ar/herramienta-web-3/el-desarrollo-humano-sustentable-no-es-posible-en-el-capitalismo-la-construccion-d (11/2009).
García, A.; Murillo, G.; Lara, M. y Rodríguez, D. 2015. Gestión sustentable de empresas agroalimentarias factores clave de estrategia competitiva. Universidad Técnica Estatal de Quevedo. Quevedo. Ecuador.

PNUD. 2013. Informe sobre desarrollo humano. El ascenso del Sur: Progreso humano en un mundo diverso. Programa de las Nacionales Unidas para el Desarrollo. Nueva York. Estados Unidos. 2016 pp. PNUD. 2014. Índice de desarrollo humano municipal en México: Nueva metodología. Programa de Naciones Unidas para el Desarrollo (PNUD). México DF. México.

Ramos, E. y Garrido, D. 2011. Desarrollo rural territorial. Metodología y aplicación para estudios de casos. Ministerio de Medio Ambiente y Medio Rural y Marino. Madrid. España.

Rangel-Quintos, J.; Espinosa, J.; De Pablos, C.; Angón, E.; Perea, J.; Rivas, J., y García, A. 2014. Indicadores de desarrollo humano en el sistema bovino de doble propósito en el trópico mexicano. Rev Cient Univ Técnica Estatal Quevedo, 7: 183-187.

Torres, Y.; Rivas, J.; De Pablos, C.; Perea, J.; Toro-Mujica, P.; Angón, E. y García, A. 2014. Identificación e implementación de paquetes tecnológicos en ganadería vacuna de doble propósito. Caso ManabíEcuador. Rev Méx Cienc Pec, 5: 393-407. 